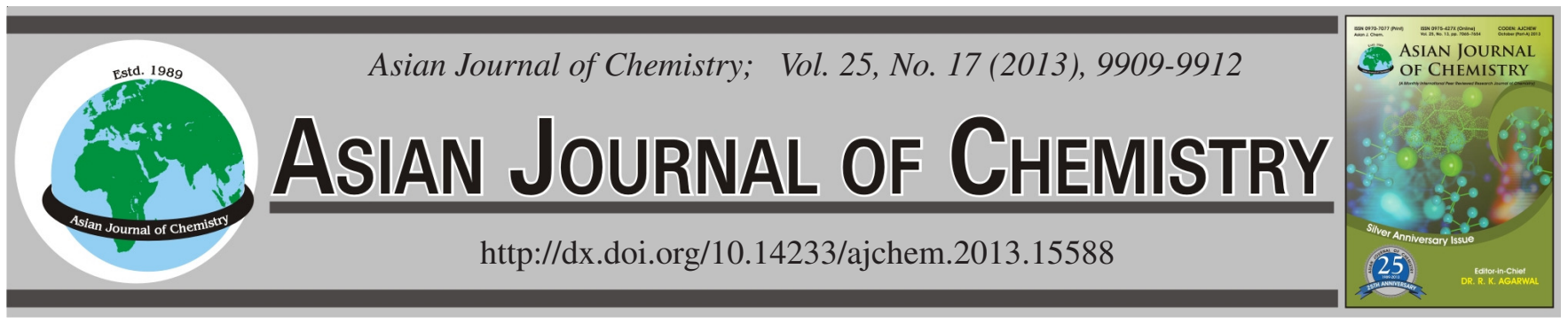

\title{
Comparative Analysis of Bind Energies and Sites Between Simvastatin and Atorvastatin into CYP3A4
}

\author{
Tie-Chao Jiang ${ }^{1}$, Ning Kong ${ }^{2}$, Shan-Shan Zhao ${ }^{3}$ and Ping Yang ${ }^{1, *}$
}

${ }^{1}$ China-Japan Union Hospital of Jilin University, Changchun 130033, Jilin Province, P.R. China

${ }^{2}$ Department of Regenerative Medicine, College of Pharmacy, Jilin University, Changchun 130021, Jilin Province, P.R. China

${ }^{3}$ State Key Laboratory of Supramolecular Structure and Materials, Jilin University, Changchun 130024, P.R. China

*Corresponding author: E-mail: pyang@jlu.edu.cn

(Received: 6 April 2013;

Accepted: 28 October 2013)

AJC-14303

\begin{abstract}
The three-dimensional (3D) models of simvastatin and atorvastatin into CYP3A4 enzyme were constructed based on the crystal structure (PDB ID:3UA1) and refined by molecular dynamics simulations. The results showed that simvastatin and atorvastatin can stably bound into the active site of CYP3A4 enzyme. The hydrogen bonds and hydrophobic interactions played an important role in the stabilities of the conformation of the complexes. The interactions between simvastatin, atorvastatin and CYP3A4, as well as anchor amino acid residues for substrates binding were discussed. In addition, the potential dehydrogenated site by CYP3A4 of simvastatin and atorvastatin site was pointed out, which was in agreement with the experiment in vivo.
\end{abstract}

Key Words: Simvastatin, Atorvastatin, Molecular docking, Molecular dynamics simulation

\section{INTRODUCTION}

At present, cardio-cerebrovascular disease has become the No. 1 killer to threaten people's health and experiment have proven that high cholesterol is an important independent risk factor. Statins are regarded as the most potent drugs used to lower cholesterol levels and are widely applied in clinic ${ }^{1}$. Statins can efficiently inhibit the 3-hydroxy-3-methylglutaryl coenzyme A (HMG-CoA) reductase, a kind of rate-limiting enzyme in the biosynthesis of cholesterol and then reduce total cholesterol and the low density lipoprotein (LDL) cholesterol levels in blood ${ }^{2,3}$. Statins have rare but severe adverse effect, such as myopathies and rhabdomyolysi ${ }^{4,5}$. Previous researches have certified that the adverse effects of statins are dose dependent and the risk is amplified by drug interactions that functionally increase statin potency, often through inhibition of the cytochrome CYP3A4 system ${ }^{6}$. Simvastatin, lovastatin, atorvastatin and cerivastatin, which are mainly metabolized in the liver by the CYP3A4, have a high potential for CYP3A4mediated drug-drug interactions (DDIs) ${ }^{7-10}$. Co-administration of CYP3A inhibitors, such as cyclosporine, itraconazole, erythromycin, grapefruit juice and diltiazem, influence the pharmacokinetic disposition of statins and then raising the risk of adverse effect ${ }^{11-15}$.

For the simvastatin and atorvastatin (Fig. 1), taking same dose itraconazole leads to the AUC values of simvastatin

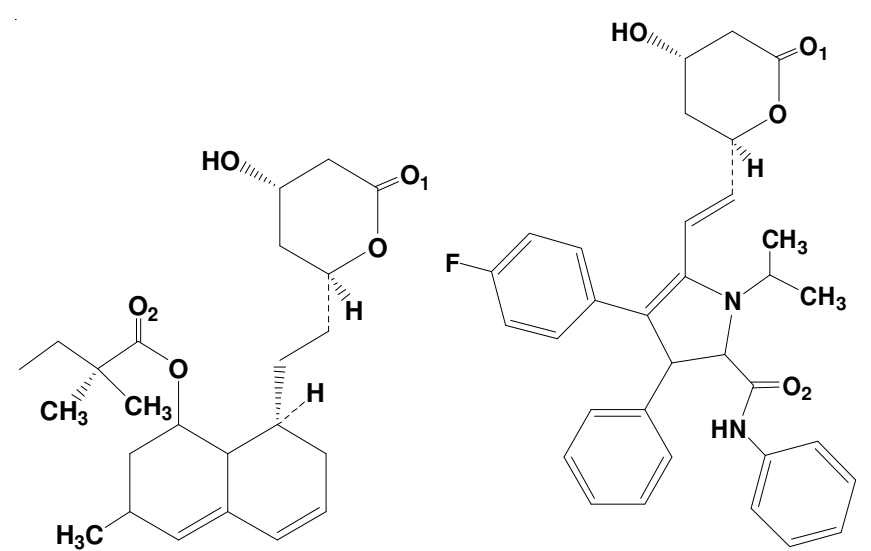

Fig. 1. Molecular structures of simvastatin (A) and atorvastatin (B)

increased by 20 times, while that of atorvastatin increased by about 3 times $^{16,17}$. Drinking $200 \mathrm{~mL}$ concentrated grapefruit juice results in the increase of AUC values by 16 times and 2.5 times for simvastatin and atorvastatin, respectively ${ }^{18,19}$. We hypothesize that the discrepancy of CYP3A4-mediated drug interactions in clinical is consider to different bind energy of simvastatin and atorvastatin to CYP3A enzymes. Recently, molecular dynamics simulation has proven useful in gaining insight into the mechanism of interaction between drug and enzyme as well as predicting the interaction value ${ }^{20-22}$. The theoretical calculations are necessary to explain experimental 
phenomena and successfully approved by a wide range of experimental measurements. In this article, we investigate the interaction between simvastatin and atorvastatin into CYP3A4, aiming at illuminating the possible reason of pharmacologic variability between simvastatin and atorvastatin.

\section{CALCULATION METHOD}

The equilibrium structures of simvastatin and atorvastatin were optimized by the density functional theory (DFT) method with the Becke three-parameter hybrid exchange and the LeeYang-Parr correlation functional (B3LYP $)^{23}$. Gradient optimizations were carried out using the $6-31 \mathrm{G}^{*}$ basis set for $\mathrm{C}, \mathrm{N}, \mathrm{F}$, $\mathrm{O}$ and $\mathrm{H}$ atoms. Harmonic vibrational frequencies were calculated at the same theoretical level based on the optimized geometries. The results showed no imaginary frequency, indicating the optimized geometries were the most stable configuration. All the calculations were performed using the Gaussian 09 software package.

The CYP3A4 initial structure was obtained from the PDB Bank database (PDB ID: 3UA1). The missing loops of the template protein were appended on the basis of relatively complete CYP3A4 protein. Autodock 4.0 software with default parameters was employed to dock simvastatin and atorvastatin into CYP3A4.

The hydrogenation of the obtained complexes was taken by VMD system. Then the proteins were solvated in a $10 \AA$ layer of TIP3P water molecules to account for solvent effect. The sodium ions and chloride ions were added in the system to ensure the electrical neutral. To further consider the flexibility of protein, molecular dynamics simulation was performed for each ligand-CYP3A4 complexes by CHARMM force field in NAMD software package ${ }^{24}$. Energy minimization of 1000 steps steepest decent (SD) followed by 300 steps conjugated gradient (CG) was carried out. Then the complexes were gradually heated from $0 \mathrm{~K}$ to $300 \mathrm{~K}$ and subjected to equilibration dynamics simulation at a constant temperature $300 \mathrm{~K}$. Then $5 \mathrm{~ns}$ molecular dynamics production runs were performed in the NPT ensemble at $101.3 \mathrm{kPa}$ and $300 \mathrm{~K}$ with time step $1 \mathrm{fs}$ and a non-bond interaction cutoff distance $1 \mathrm{~nm}$. The average complex structures from the molecular dynamics simulations were taken to discuss the interaction energy in the following section.

\section{RESULTS AND DISCUSSION}

Electronic structures: For the frontier molecular orbitals (FMOs), namely the highest occupied molecular orbital (HOMO) and the lowest unoccupied orbit (LUMO), are more relative to the redox properties. The distributions of FMOs in simvastatin and atorvastatin are presented in this work. The results based on the B3LYP function with 6-31G* basis set are presented in Fig. 2. It is shown that the HOMO and LUMO of simvastatin and atorvastatin possess delocalized $\pi$-orbital features. For the simvastatin, the $\pi$ orbital of methyl substituted on the hexahydro-naphthalene contributes primarily to the HOMO and LUMO, indicating that the methyl behave higher reactivity and prone to oxidation reduction reaction. For atorvastatin, the HOMO and LUMO are mostly located on the central five-member heterocycle.
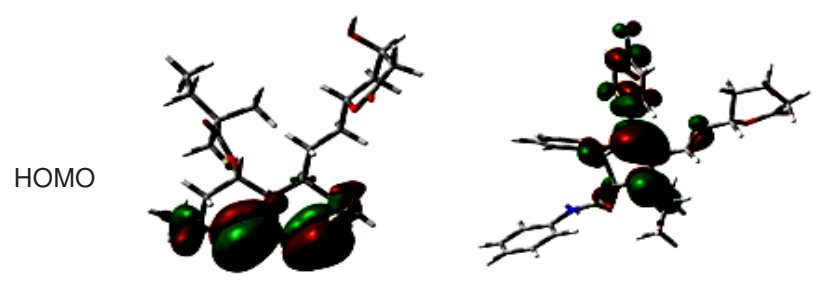

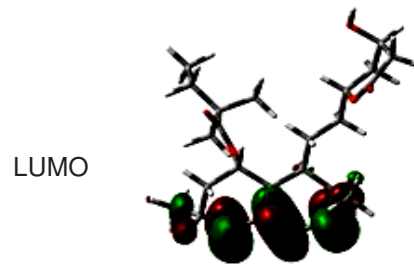

Simvastatin
Atorvastatin

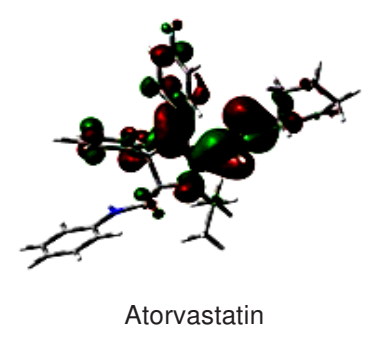

Fig. 2. Structures of simvastatin and atorvastatin present as tube-type. The frontier molecular orbitals of simvastatin and atorvastatin calculated at B3LYP wave function

Molecular docking and dynamics simulations: CYP3A4 is one of the most famous enzymes in CPY450 because of its broad substrate specificity, such as immune inhibitors, calcium channel blockers, cancer chemotherapy drugs and other 150 kinds of drugs. CYP3A4 exists mainly in human liver and accounts approximately for about $30 \%$ of total CPY 450. Williams et al. ${ }^{25}$ and Yano et al. ${ }^{26}$ have devoted their efforts to investigate the structure of CYP3A4. Several CYP3A4 crystals with apo or holo structures are available in the PDB database. The conformation and size of the active pocket of CYP3A4 can be induced by the ligand. Considering to the size of simvastatin and atorvastatin, we selected the crystal structure (PDB ID: 3UA1) to do the docking studies. The combination of Lamarckian genetic algorithm and empirical binding free energy function in Autodock 4.0 was used to search for the optimal orientation of simvastatin and atorvastatin into CYP3A4. Semi-flexible docking was adopted, that were only the binding site atoms and ligand atoms are allowed to move during the docking process. Then $5 \mathrm{~ns}$ molecular dynamics simulations for simvastatin-CPY3A4 and atorvastatinCPY3A4 complexes were performed to fully consider the flexibility of protein and ligand. The root mean square deviation (RMSD) reached equilibrium after 5 ns molecular dynamics simulations for these complexes. It meant that simvastatin and atorvastatin stably bound into CYP3A4 (Fig. 3).

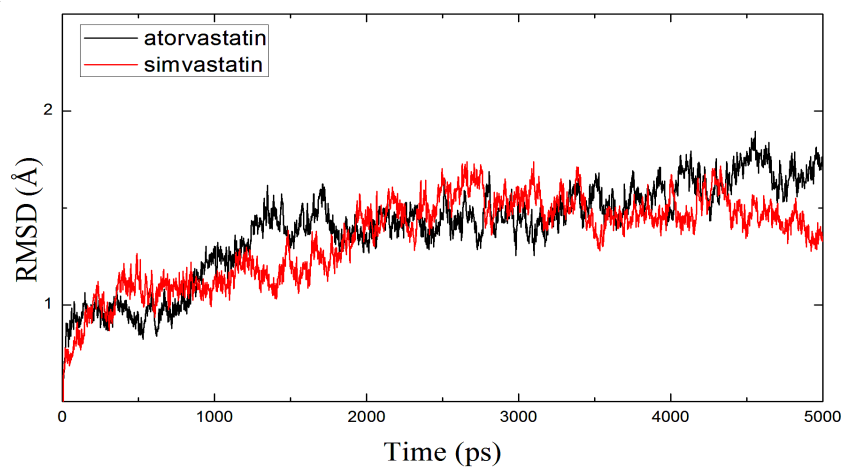

Fig. 3. Variations of Ca RMSD during 5 ns MD simulations of complexes: simvastatin-CYP3A4 and atorvastatin-CYP3A4 
Bind energy of simvastatin and CYP3A4: The binding mode of simvastatin and CYP3A4 is shown in Fig. 4. In order to identify the importance of amino acid residues in the active pocket and evaluate its values, we calculated the interaction energies between amino acid residues and the simvastatin. The selected interaction energies are lower than $-0.1 \mathrm{~kJ} / \mathrm{mol}$ (Table1). The results indicated that simvastatin are stably positioned into the active site of CYP3A4 with the favorable total interaction energy of $-77.09 \mathrm{~kJ} / \mathrm{mol}$. The van der Waals energy and electrostatic energy were $-45.59 \mathrm{~kJ} / \mathrm{mol}$ and $-35.91 \mathrm{~kJ} / \mathrm{mol}$, which mainly stabilized the complex. Two hydrogen bonds contributed to the interaction between simvastatin and the CYP3A4 enzyme: one was the hydrogen bond formed by the $\mathrm{O}_{2}$ atom of carbonyl and the ARG212 residue, the other was the one between $\mathrm{O}_{1}$ atom of carbonyl and ARG106 residue. Additionally, the hydrophobic between ARG372, PHE108, ASP214, PHE213, PHE215 and the ester of six-member ring also contributed much the stabilization energy. In the complex, the methyl C atom is $4.26 \AA$ far from the Fe atom in the heme. Generally, the Fe atom in the heme can display catalytic role and the catalytic capability is completely related to distance between reaction sites. In the simvastatin-CYP3A4 complex,

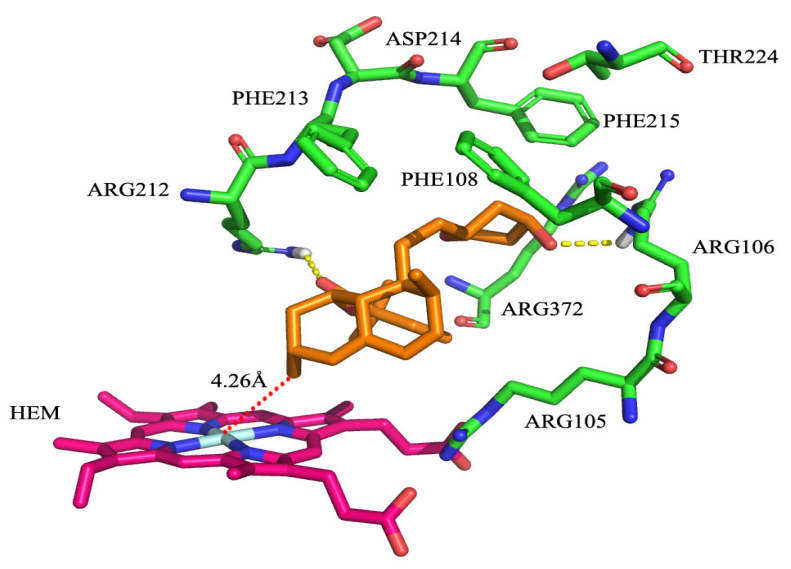

(a)

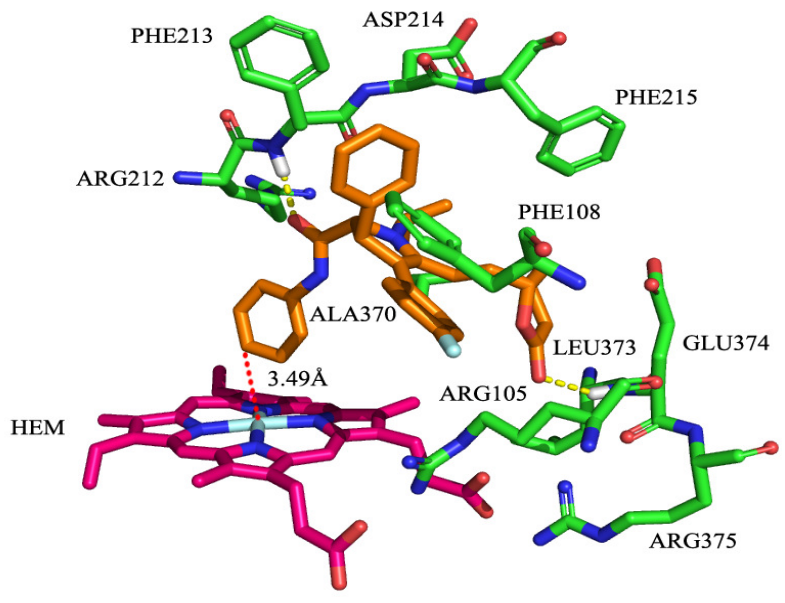

(b)

Fig. 4 Binding mode of simvastatin-CYP3A4 (a) and atorvastatin-CYP3A4 (b). The heme group is represented by a red stick. The hydrogen bonds are shown in yellow dashed lines. The distance between Fe atom in heme and the adjacent $\mathrm{C}$ atoms are shown in red dashed lines

\begin{tabular}{lccc}
\multicolumn{5}{c}{ TABLE-1 } \\
\multicolumn{4}{c}{ TOTAL ENERGY $\left(\mathrm{E}_{\text {total }}\right)$, VAN-DER-WAALS ENERGY $\left(\mathrm{E}_{\text {vdw }}\right)$} \\
AND ELECTROSTATIC ENERGY $\left(\mathrm{E}_{\text {ele }}\right.$ ) OF SIMVASTATIN \\
AND INDIVIDUAL RESIDUES IN CYP3A4 \\
\hline Simvastatin & $\mathrm{E}_{\text {vdw }}(\mathrm{kJ} / \mathrm{mol})$ & $\mathrm{E}_{\text {ele }}(\mathrm{kJ} / \mathrm{mol})$ & $\mathrm{E}_{\text {total }}(\mathrm{kJ} / \mathrm{mol})$ \\
\hline Total & -45.59 & -35.91 & -77.09 \\
ARG 212 & -3.40 & -15.88 & -19.28 \\
HEM508 & -5.02 & -2.68 & -7.70 \\
ARG 106 & -3.91 & -3.30 & -7.21 \\
ASP 214 & -0.92 & -5.90 & -6.82 \\
ARG 372 & -1.43 & -3.79 & -5.22 \\
PHE 213 & -3.36 & -1.09 & -4.45 \\
ARG 105 & -4.60 & 0.50 & -4.10 \\
THR 224 & -1.51 & -2.46 & -3.97 \\
PHE 215 & -2.63 & -1.12 & -3.75 \\
PHE 108 & -3.58 & 0.13 & -3.45 \\
SER 119 & -1.78 & -0.83 & -2.61 \\
ILE 120 & -1.95 & -0.16 & -2.11 \\
PHE 304 & -1.92 & 0.28 & -1.64 \\
ILE 57 & -0.96 & -0.64 & -1.60 \\
GLY 481 & -2.12 & 0.86 & -1.26 \\
LEU 373 & -1.16 & 0.13 & -1.03 \\
ILE 301 & -0.29 & 0.02 & -0.27 \\
THR 433 & -0.01 & -0.25 & -0.26 \\
ALA 305 & -4.9 & 0.27 & -0.22 \\
VAL 240 & -0.14 & 0.00 & -0.14 \\
\hline
\end{tabular}

the methyl $\mathrm{C}$ atom is localized at a reasonable distance from $\mathrm{Fe}$ atom as a reaction site. This result is in agreement with the experimental data that is the methyl $\mathrm{C}$ atom could form an electrophilic intermediate and then conduct hydroxylation reaction ${ }^{27}$.

Bind energy of atorvastatin and CYP3A4: For atorvastatinCYP3A4 complex, the interaction energies of atorvastatin and each residue in the active site were calculated. Residues with the interaction energy lower than $-0.4 \mathrm{~kJ} / \mathrm{mol}$ are shown in Table-2. The results showed that the interaction of atorvastatin and CYP3A4 is much stronger than that of simvastatin and CYP3A4. The total interaction energy is $-93.81 \mathrm{~kJ} / \mathrm{mol}$

TABLE-2

TOTAL ENERGY ( $\left.\mathrm{E}_{\text {total }}\right)$, VAN-DER-WAALS ENERGY ( $\left.\mathrm{E}_{\mathrm{vdW}}\right)$ AND ELECTROSTATIC ENERGY ( $\left.\mathrm{E}_{\mathrm{ele}}\right)$ OF ATOR- VASTATIN AND INDIVIDUAL RESIDUES IN CYP3A4

\begin{tabular}{lccc}
\hline Atorvastatin & $\mathrm{E}_{\mathrm{vdW}}(\mathrm{kJ} / \mathrm{mol})$ & $\mathrm{E}_{\text {ele }}(\mathrm{kJ} / \mathrm{mol})$ & $\mathrm{E}_{\text {total }}(\mathrm{kJ} / \mathrm{mol})$ \\
\hline Total & -46.97 & -46.84 & -93.81 \\
GLU 374 & -3.99 & -8.63 & -12.62 \\
ARG 105 & -3.68 & -8.40 & -12.08 \\
PHE 213 & -5.37 & -4.93 & -10.30 \\
ALA 370 & -1.89 & -7.56 & -9.45 \\
ARG 212 & -3.43 & -5.25 & -8.68 \\
ARG 375 & -0.38 & -6.42 & -6.80 \\
ASP 214 & -2.67 & -4.04 & -6.71 \\
PHE 108 & -4.12 & -1.05 & -5.17 \\
LEU373 & -2.19 & -2.53 & -4.72 \\
PHE 215 & -3.73 & 0.46 & -3.27 \\
PHE304 & -2.74 & 0.39 & -2.35 \\
PHE 220 & -2.13 & -0.20 & -2.33 \\
ARG 440 & -0.17 & -2.05 & -2.22 \\
ALA 305 & -1.31 & -0.55 & -1.86 \\
ARG 372 & -2.47 & 1.31 & -1.16 \\
THR 309 & -0.68 & -0.30 & -0.98 \\
ILE 120 & -2.04 & 1.11 & -0.93 \\
SER 119 & -1.61 & 0.81 & -0.80 \\
VAL 240 & -0.91 & 0.42 & -0.50 \\
LEU 482 & -0.44 & -0.02 & -0.46 \\
PHE 57 & -1.02 & 0.59 & -0.42 \\
\hline
\end{tabular}


including $-46.84 \mathrm{~kJ} / \mathrm{mol}$ of electrostatic energy and $-46.97 \mathrm{~kJ} /$ mol of van der Waals energy. Similar to the simvastatin, atorvastatin also formed two hydrogen bonds with CYP3A34. They are the ones between the carbonyl $\mathrm{O}$ atoms and the $\mathrm{H}$ atoms of the neighboring PHE213 and GLU374 residues, respectively. Meanwhile, the hydrophobic interactions between the hydrophobic cluster PHE213, ARG212 ASP214 and ARG105, LEU373, PHE108 further stabilized atorvastatin in CPY3A4 active pocket. In this complex, the Fe atom was adjacent to the benzene ring with the shortest distance of $3.49 \AA$ (Fig. 3), indicating that this $\mathrm{C}$ atom prone to the dehydrogenation reaction. It is consistent with the experimental results ${ }^{28}$.

\section{Conclusion}

In this paper, molecular docking and molecular dynamics simulations were performed to investigate the three-dimensional structure of simvastatin-CYP3A4 and atorvastatinCYP3A4 complexes. The results showed that simvastatin and atorvastatin stably bound into active pocket of CYP3A4. The driving force was the hydrogen bonding and hydrophobic interactions. Compared with simvastatin, atorvastatin showed stronger binding capacity to CYP3A4. Thus the impact of CYP3A inhibitors on atorvastatin pharmacoki-netics is significantly smaller than that of simvastatin. That is why the drug interactions discrepancy of simvastatin and atorvastatin caused during the CYP3A4-mediated precess. It is in well agreement with the clinical results that atorvastatin is more non-sensitive to the CYP3A4-mediated drug. Though the bond energy calculated in our paper is limited, hence, we believe that it is accurate enough to justify the qualitative conclusions. The MD simulations of statin drugs play an important role in predicting the activity and pharmacological mechanism of station drugs, which provides theoretical evidence to clinical work.

\section{REFERENCES}

1. J. Kidd, Nat. Rev. Drug Discov., 5, 813 (2006).

2. A. Corsini, F.M. Maggi and A.L. Catapano Pharmacol. Res., 31, 9 (1995).

3. P. Libby, M. Aikawa and U.K. Schonbeck, Biochim. Biophys. Acta, 1529, 299 (2000).

4. J.A. Staffa, J. Chang and L. Green, N. Engl. J. Med., 346, 539 ( 2002).
5. P.D. Thompson, P. Clarkson and R.H. Karas, J. Am. Med. Assoc., 289, 1681 (2003).

6. K. Kajinami, H. Masuya and Y. Hoshiba, Expert. Opin. Pharmacother., 61291 (2005).

7. A. Suri, W.P. Forbes and S.L. Bramer, Clin Pharmacokinet, 37, 61 (1999).

8. R.F. Reinoso, N.A. Sanchez and M.J. Garcia, Methods Find Exp. Clin. Pharmacol., 23, 541 (2001).

9. C. de Graaf, N.P. Vermeulen and K.A. Feenstra, J. Med. Chem., 48, 2725 ( 2005)

10. I.G. Denisov, T.M. Makris and S.G. Sligar, Chem. Rev., 105, 2253 (2005).

11. D.G. Le Couteur, P.T. Martin and P. Bracs, Proc. Aust. Soc. Clin. Exp. Pharmacol. Toxicol., 3, 153 (1996).

12. A.E. Black, R.N. Hayes, B.D. Roth, P. Woo and T.F. Woolf, Drug Metab. Dispos., 27, 916 (1999).

13. H. Lennernas, Clin. Pharmacokinet., 42, 1141 (2003).

14. M.B. Bottorff, Am. J. Cardiol., 97, 27C (2006).

15. C.G. Rowan, S.M. Brunelli, J. Munson, J. Flory, P.P. Reese, S. Hennessy, J. Lewis, D. Mines, J.S. Barrett, W. Bilker and B.L. Strom, Pharmacoepidemiol. Drug Saf., 21, 494 (2012).

16. T. Hulgan, T.R. Sterling, I. Daugherty, P.G. Arbogast, S. Raffanti and W. Ray, J. Acquir. Immune Defic. Syndr., 38, 277 (2005).

17. T. Kantola, K.T. Kivisto and P.J. Neuvonen, Clin. Pharmacol. Ther., 64, 58 (1998)

18. T. Kantola, K.T. Kivisto and P.J. Neuvonen, Clin. Pharmacol. Ther., 63, 397 (1998).

19. J.J. Lilja, K.T. Kivisto and P.J. Neuvonen, Clin. Pharmacol. Ther., 66, 118 (1999).

20. J.L. Zhang, Q.C. Zheng, Z.Q. Li and H.X. Zhang, PLOS One, 7, e39546 (2012).

21. X.Y. Meng, Z. Li, R.J. Niu, H.X. Zhang and Q.C. Zheng, Chem. Res. Chin. Univ., 28, 137 (2012).

22. W.T. Chu, Y.J. Wu, J.L. Zhang, Q.C. Zheng, L. Chen, Q. Xue and H.X. Zhang, Biochim. Biophys. Acta., 7, 913 (2012).

23. P.J. Stephens, F.J. Devlin and C.F. Chabalowski, J. Phy. Chem., 98, 11623 (1994).

24. S.E. Feller and A.D. MacKerell Jr., J. Phys. Chem. B, 104, 7510 (2000).

25. P.A. Williams, J. Cosme, D.M. Vinkovic, A. Ward, H.C. Angove, P.J. Day, C. Vonrhein, I.J. Tickle and H. Jhoti, Science, 305, 683 (2004).

26. J.K. Yano, M.R. Wester, G.A. Schoch, K.J. Griffin, C.D. Stout and E.F. Johnson, J. Biol. Chem., 279, 38091 (2004).

27. K.H. Kim, J.Y. Kang, D.H. Kim, S.H. Park, S.H. Park, D. Kim, K.D. Park, Y.J. Lee, H.C. Jung, J.G. Pan., T. Ahn and C.H. Yun, Drug Metab. Dispos., 39, 140 (2011).

28. W. Jacobsen, B. Kuhn, A. Soldner, G. Kirchner, K.F. Sewing, P.A. Kollman, L.Z. Benet and U. Christians, Drug Metab. Dispos., 28, 1369 (2000). 